\title{
Exkurs: Unsicherheiten
}

\section{bei der Analyse und Attribution}

von Hochwasserereignissen

Manfred Mudelsee

11.1 Elbehochwasser - 104

11.2 Unsicherheiten bei der statistischen Analyse von

Ereignissen in der Vergangenheit - 105

11.3 Unsicherheiten bei der statistischen Analyse von Projektionen in die Zukunft - 107

11.4 Unsicherheitsreduzierung durch intelligentes Fragen - 108

$11.5 \quad$ Kurz gesagt -108

Literatur - 109 
Extremereignisse zeigen am augenfälligsten, wie verletzlich Deutschland gegenüber dem Klima und seinen Veränderungen ist. Betrachtet man Extremereignisse genauer, verursachten in den vergangenen 20 Jahren Hochwasser die größten Schäden (Ernst Rauch, Münchener Rückversicherungs-Gesellschaft, persönliche Mitteilung). In der Wissenschaft herrscht Einigkeit darüber, dass sich der zukünftige globale Wasserkreislauf durch steigende atmosphärische Treibhausgaskonzentrationen verändern wird (Kirtman et al. 2013). Doch selbst bei der vergleichsweise guten Datenlage für Deutschland ist es unsicher, ob sich die Auftrittsrate - die Anzahl an Ereignissen pro Jahr - von Hochwasser verändert (Trend), wie stark eventuell vorliegende Trends sind und wie stark der Klimawandel ursächlich einwirkt. Diese Zuschreibung der Ursachen wird als Attribution bezeichnet.

Gleichzeitig bilden diese Informationen eine wichtige Grundlage für Entscheidungsträger, die über Mitigations- und Anpassungsstrategien befinden. Die damit verbundenen Unsicherheiten müssen daher möglichst transparent kommuniziert werden, um einen Umgang damit zu ermöglichen. Ihre Quellen und Ausmaße werden im Folgenden am Beispiel der Elbehochwasser ausführlich illustriert. Für die Elbe ist der Wissensstand aufgrund der guten Datenqualität und umfangreicher wissenschaftlicher Untersuchungen relativ hoch. Für andere Flüsse ( Kap. 10) und andere Ereignistypen sind die Unsicherheiten zum Teil wesentlich größer.

\subsection{Elbehochwasser}

Das Hochwasser in Zentraleuropa im Juni 2013 verursachte direkte ökonomische Schäden in Höhe von insgesamt 11,7 Mrd. Euro bzw. 10,0 Mrd. Euro in Deutschland und das Hochwasser im August 2002 Schäden in Höhe von insgesamt 16,7 Mrd. Euro bzw. 11,6 Mrd. Euro in Deutschland (Ernst Rauch, Münchener Rückversicherungs-Gesellschaft, persönliche Mitteilung). Einige Menschen verloren bei diesen Ereignissen ihr Leben. Beide Male war das Gebiet der Elbe besonders betroffen.

Die beste, d.h. die genaueste und zeitlich längste Datengrundlage über Elbehochwasser ist die Abflusszeitreihe vom Pegel Dresden (-Abb. 11.1). Abfluss ist definiert als Volumen Wasser pro Zeit. Die Abflusswerte sind abgeleitet über Eichbeziehungen aus den gemessenen Wasserständen. Dies stellt eine Quelle von Unsicherheiten dar, weil 1) Messwerte grundsätzlich immer fehlerbehaftet sind (Ablesefehler, Geräteungenauigkeiten) und 2) - im vorliegenden Fall gravierender - die Form der Eichkurve nicht physikalisch abgeleitet, sondern nur empirisch bestimmt werden kann ( Abschn. 11.2). Die Daten zeigen, dass die Hochwasser im August 2002 und Juni 2013 zu den größten in Dresden gemessenen Ereignissen gehören (Conradt et al. 2013), aber auch frühere Elbehochwasser (z. B. im März/April 1845) waren von vergleichbarer Größenordnung (Königliche Elbstrombauverwaltung 1898).

Das Ergebnis der Analyse der Hochwasserwahrscheinlichkeit (- Abb. 11.2) zeigt beträchtliche Unsicherheitsbereiche der geschätzten Hochwasserauftrittsraten über die vergangenen 200 Jahre. Es wird saisonal differenziert, da die Ursachen von
Hochwasser im Winter (von der Hydrologie festgesetzt auf November bis April) sich von denen für den Sommer unterscheiden. Der Fokus in der räumlichen Dimension liegt auf der mittleren Elbe (zwischen den Städten Litoměřice und Magdeburg). „Geschätzt" bezieht sich dabei auf die Auftrittsratenkurve, die bei der vorliegenden Datenmenge und bei Gültigkeit der gemachten Annahmen (• Tab. 11.1) idealerweise nur wenig von der wahren, jedoch unbekannten Auftrittsratenkurve abweicht. „90\%-Band“ bedeutet, dass für einen einzelnen Zeitpunkt die unbekannte Auftrittsrate mit einer Wahrscheinlichkeit von $90 \%$ zwischen der oberen und der unteren Bandgrenze liegt. Beispiel: Das Maximum der Auftrittsrate von Winterhochwasser von 0,22 pro Jahr (im Mittel ein Ereignis alle 4-5 Jahre) im Jahr 1832 liegt zu $90 \%$ zwischen 0,13 und 0,31 pro Jahr. Auch die Korrektheit des Bandes hängt von der Gültigkeit der getroffenen Annahmen (- Tab. 11.1) ab. Wie stark die Effekte sind, die auftreten, wenn diese Annahmen nicht zutreffen, lässt sich im Prinzip mithilfe von Sensitivitätsanalysen bestimmen. $\mathrm{Zu}$ weiteren Details der Analyse, Attribution und Interpretation siehe Abschn. 11.2 und Mudelsee et al. (2003, 2004).

Es zeigt sich, dass Winterhochwasser der mittleren Elbe längerfristig - d.h. auf Zeitskalen von mindestens drei Jahrzehnten - seltener werden und Sommerhochwasser im Auftreten konstant bleiben (- Abb. 11.2). Winterhochwasser traten im Mittel über den Zeitraum 1806-2002 etwa 6,4-mal häufiger auf als Sommerhochwasser. Für kürzere Zeitskalen - also weniger als drei Jahrzehnte - sind keine belastbaren Aussagen möglich, da die Datenanzahl zu gering und der statistische Fehler zu groß ist (- Tab. 11.1).

Die beobachteten Trends lassen sich zum Teil attributiv dem Klimawandel zuordnen. Eine Attribution hat grundsätzlich den Charakter einer Hypothese, die mithilfe der Daten getestet wird und deren Ergebnis eine Irrtumswahrscheinlichkeit aufweist. Die beobachtete regionale Erwärmung im Einzugsgebiet (Hartmann et al. 2013) lässt den Fluss winters seltener vereisen und reduziert damit die Wahrscheinlichkeit des Auftretens von „Eishochwasser“. Hierbei führt eine aufbrechende Eisdecke zu einer Verstauung der Schollen, was eine örtliche Wasserstandserhöhung bewirkt, wie z. B. im März/April 1845. Die Attribution für Sommerhochwasser, die durch Extremniederschläge ausgelöst werden, erfolgt über eine andere Kausalkette. Regionale Erwärmung führt zu einer steigenden atmosphärischen Wasserdampfaufnahmefähigkeit, die eine erhöhte Auftrittsrate extremer Niederschläge und damit eine erhöhte Hochwasserauftrittsrate zur Folge hat (Allen und Ingram 2002; Boucher et al. 2013; Hirabayashi et al. 2013). Diese Kette ist jedoch deutlichen Störeinflüssen ausgesetzt, weshalb eventuell vorhandene Trends für den Zeitraum in • Abb. 11.2 (bis August 2002) nicht nachweisbar sind (Mudelsee et al. 2004). Störeinflüsse könnten hypothetisch in den Änderungen der am häufigsten auftretenden Windrichtungen und des damit zusammenhängenden orografischen Niederschlags (Mudelsee et al. 2006) bestehen. Die bewegten Luftmassen werden hierbei an den Gebirgen gehoben und dadurch abgekühlt, und der gasförmige, in der Luft enthaltene Wasserdampf kondensiert in flüssigen Niederschlag. Das ist ein Störeinfluss, weil von einer signifikanten physikalischen Einwirkung auszugehen ist, die jedoch in ihrer Größe nicht ge- 
nau bekannt ist, weil Windrichtungen räumlich und zeitlich stark schwanken. Möglicherweise sind mittlerweile Trends im Auftreten von Sommerhochwasser der Elbe nachweisbar, da mehr Ereignisse vorliegen; dazu liegt jedoch noch keine begutachtete Literatur vor. Die Bestimmung des maximalen Abflusses am Hochwasserscheitel im Juni 2013 wurde erst Anfang 2014 abgeschlossen.

Die Befunde sind relativ robust. Das bedeutet in diesem Fall: kaum beeinflusst durch die Wahl des Schwellenwerts für die Definition extrem großer Ereignisse (- Abb. 11.1), wie Sensitivitätsanalysen (Mudelsee et al. 2004) zeigen. Sensitivitätsanalysen sind Computerexperimente, die untersuchen, wie sich das Ergebnis ändert, wenn man die zugrunde liegenden Annahmen ändert (Mudelsee 2014). Beispielsweise ergeben sich auch abfallende Trends im Auftreten von Winterhochwasser der Elbe, wenn man den Schwellenwert auf die Grenze für kleinere Hochwasser herabsetzt (Mudelsee et al. 2003). Ein statistischer Test erhärtet diese Trendbefunde (Mudelsee et al. 2004).

Eine Anzahl alternativer Erklärungen für vorliegende Trends ist prinzipiell denkbar. Zu diesen zählen Wasserbau und Landnutzungsänderungen.

Im Wasserbau kann ein koordiniertes Management der Hochwasserrückhaltebecken und anderer Reservoire den Hochwasserscheitel senken, wie für die Elbe im August 2002 bei Magdeburg (Bronstert 2003) und im Juni 2013 bei mehreren Stationen (Belz et al. 2013) gezeigt wurde. Hier zählt jeder Zentimeter, weil die Kurve der Schäden gegenüber der Ereignisstärke, wie generell bei vielen Ereignistypen, im oberen Bereich sehr stark ansteigt (Stern 2007; Ward et al. 2011). Sensitivitätsanalysen zeigen jedoch, dass die Auftrittsraten extrem starker Hochwasser der mittleren Elbe dadurch nicht reduziert werden können (Mudelsee et al. 2004). Der Einfluss von Begradigungen auf die Auftrittsrate über die vergangenen drei Jahrhunderte ist vernachlässigbar (Mudelsee et al. 2003).

Auch das Argument der Landnutzungsänderungen als Ursache für die Veränderungen wurde geprüft. Die vorgetragene Behauptung (van der Ploeg und Schweigert 2001) einer Zunahme der Hochwasserauftrittsrate der unteren mittleren und unteren Elbe aufgrund von Kultivierungsmaßnahmen in der frühen DDR wurde widerlegt (Mudelsee et al. 2004):

1. Die Frist von 1949 bis vor 1989 ist zu kurz, um belastbare Ergebnisse zu erzielen.

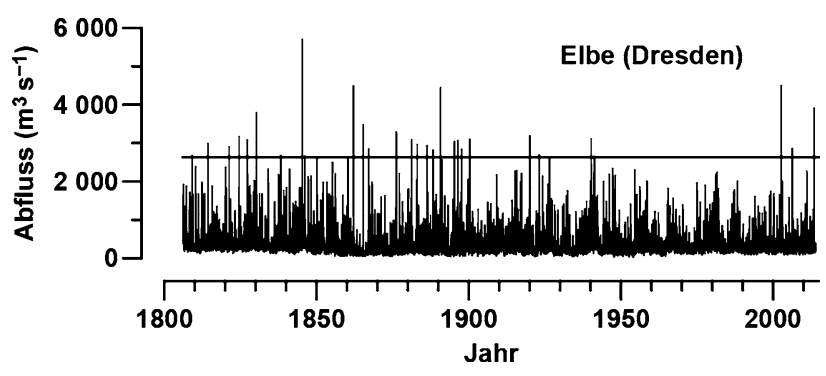

- Abb. 11.1 Abflusszeitreihe der Elbe vom Pegel Dresden für das Intervall von Januar 1806 bis Dezember 2013 (Tageswerte). Der Schwellenwert für die Definition extrem großer Ereignisse (horizontale Linie) liegt bei $2630 \mathrm{~m}^{3} \mathrm{~s}^{-1}$. (Mudelsee et al. 2003; Wasser- und Schifffahrtsverwaltung des Bundes, WSV, bereitgestellt durch die Bundesanstalt für Gewässerkunde, BfG)

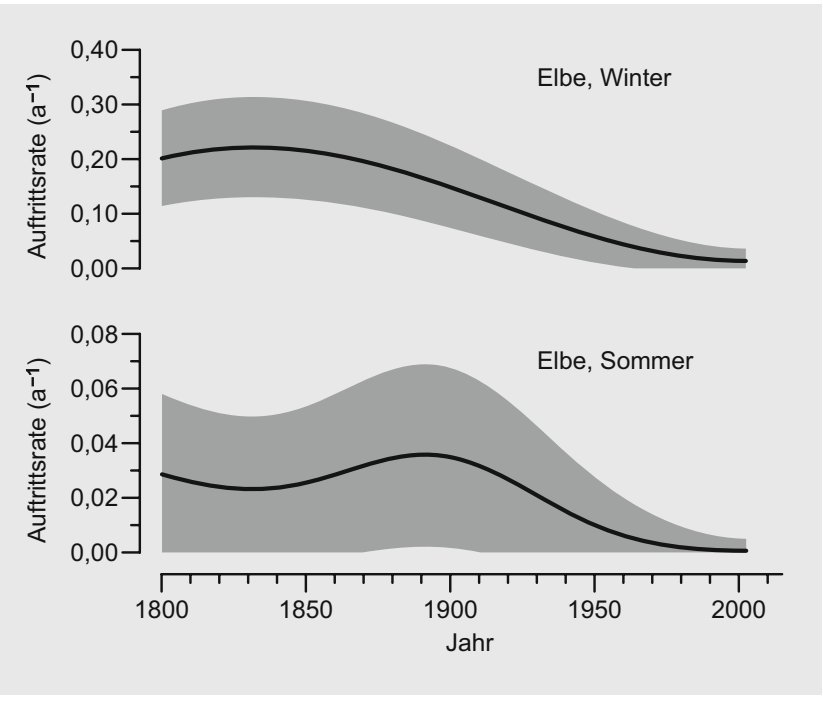

- Abb. 11.2 Geschätzte Auftrittsraten (durchgezogene Linien) extrem großer Hochwasserereignisse der mittleren Elbe, saisonal differenziert. Ebenfalls gezeigt (schattiert) sind die 90\%-Bänder der statistischen Unsicherheiten, die durch Simulationsrechnungen bestimmt wurden. (Mudelsee et al. 2003)

2. Die längerfristigen Trends weisen in die andere Richtung (• Abb. 11.2).

3. Es fehlte die saisonale Differenzierung in der genannten Arbeit.

Die grundsätzliche Herausforderung bei der Attribution ist es, zwischen unterschiedlichen Kausaleinflüssen oder Kausalkombinationen zu entscheiden. Das physikalische Vorwissen hilft, Kausaleinflüsse zu identifizieren, und die statistische Methodik hilft, diese hypothetischen Einflüsse zu testen. Wir finden im Beispiel der Elbehochwasser Hinweise auf den Attributionsfaktor „Klimawandel“ und keine Hinweise auf die Faktoren „Wasserbau“ oder "Landnutzungsänderungen“.

\subsection{Unsicherheiten bei der statistischen Analyse von Ereignissen in der Vergangenheit}

Die Auftrittsrate, die für die Analyse von Veränderungen im Hochwassergeschehen als relevante Größe betrachtet wird, ist definiert als die Anzahl an Extremereignissen pro Jahr. Bei einem sich mit der Zeit ändernden Klima kann davon ausgegangen werden, dass sich auch die Auftrittsrate ändert. Diese Zeitabhängigkeit der Auftrittsrate kann neben dem Klimawandel auch durch andere zeitabhängig einwirkende Kausalfaktoren hervorgerufen werden.

Zur Analyse der zeitabhängigen Auftrittsrate von Klimaextremen wird eine statistische Methode auf eine Datenbasis (Beobachtungen oder Modellresultate) angewendet. Die Methode besteht im Prinzip aus dem Abzählen von Ereignissen pro Zeitintervall; dazu kommen mathematische Verfeinerungen (Mudelsee 2014). Das Abzählen kann man mathematisch als eine Gewichtung beschreiben, bei der die Ereignisse innerhalb eines Abzählzeitintervalls voll, die Ereignisse außerhalb überhaupt nicht mitgerechnet werden. 
- Tab. 11.1 Unsicherheiten bei der statistischen Analyse von Hochwasser (vergangenes Klima)

\author{
Zufällige Fluktuationen \\ Ablesefehler Wasserstand* \\ Messfehler Abfluss (Eichung Wasserstand - Abfluss) \\ Kurzfristige Fluktuationen (Niederschlag) \\ Räumliche Fluktuationen (Abflussbedingungen Boden) \\ Messfehler Indikatorvariablen an natürlichen Klimaarchiven** (vor- \\ instrumentelle Periode, d. h. vor ca. $1850^{* * *}$ )
}

Begrenzte Datenverfügbarkeit (statistische Methode)

Begrenzte Rechenkapazität (statistische Methode)*

* Vernachlässigbar gering

** Zum Beispiel Seesedimente (Czymzik et al. 2010)

*** Die instrumentell gemessenen Abflusswerte für die Elbe bei Dresden (• Abb. 11.1) reichen ungewöhnlich weit (bis in das Jahr 1806) zurück

\section{Systematische Fehler}

Nullpunktsetzung Wasserstand*

Funktionale Form Eichkurve

Nichtaktualisierte Eichkurve (z. B. nach Hochwasserereignissen)

Räumliche Extrapolationen (z. B. Nebenflüsse)

Über-/Untertreibungen in historischen Klimadokumenten

Systematische Fremdeinflüsse (d. h. Einflüsse von anderen Faktoren als Hochwasser) auf Indikatorvariablen (vorinstrumentelle Periode)

Fremdeinfluss Landnutzungsänderung

Fremdeinfluss Wasserbau

Schwellenwert für die Definition extrem großer Ereignisse (statistische Methode)
Weniger „sprunghafte“ Gewichtungen, wie in - Abb. 11.2 verwendet, liefern jedoch ein genaueres Schätzergebnis (Mudelsee 2014).

Die statistische Analyse ist geprägt durch zwei Unsicherheitstypen: 1) zufällige Fluktuationen in den Beobachtungen, aber auch im komplexen Klimasystem und 2) systematische Fehler, die auftreten, wenn getroffene Annahmen nicht zutreffen. Am Beispiel der Elbehochwasser zeigt sich, dass der - zufällige - Ablesefehler des Wasserstands gegenüber den anderen Unsicherheiten vernachlässigbar gering ist. Auch der Nullpunkt des Pegels ist sehr genau bekannt. Nennenswerte Fehler können lediglich bei der Untersuchung historischer Hochwasserereignisse auftreten, wenn etwa das Datum einer Nullpunktverlegung unbekannt ist. Dies wäre ein systematischer Fehler: Eine unberücksichtigte Nullpunktsenkung etwa würde eine erhöhte Auftrittsrate für den Zeitraum danach vortäuschen.

Die hydrologisch relevante Größe für die hier vorgestellte Analyse ist der Abfluss, der jedoch aus Kostengründen nicht wie der Wasserstand kontinuierlich, sondern nur zu bestimmten Zeitpunkten gemessen wird. An die Messwertepaare Wasserstand-Abfluss wird eine Eichkurve (Mudelsee et al. 2003) angepasst. Im kontinuierlichen Betrieb wird der Abfluss aus Wasserstandsmessung und Eichkurve gewonnen. Wichtig für die Genauigkeit des Abflusswertes ist hierbei die Korrektheit der Eichkurve. Hochwasserereignisse können die Geometrie eines Flusses und damit die Form der Eichkurve jedoch verändern. Deshalb sind nichtaktualisierte Eichkurven eine Quelle systematischer Fehler (- Tab. 11.1). Die Unsicherheitstypen werden am Beispiel des Ereignistyps „Hochwasser“ dargestellt. Für die Elbe ist die Aktualisierung allerdings regelmäßig erfolgt und der entsprechende Fehler gering (Helms et al. 2002).

Selbst wenn die Abflusswerte fehlerfrei wären, würde sich keine regelmäßige Folge (z. B. jedes vierte Jahr) von Hochwasserereignissen einstellen. Deren Auftreten schwankt unregelmäßig, weil das „erzeugende“ atmosphärische System - der Niederschlag - variiert. Es ist wahrscheinlich, dass diese durch die Kli- mavariationen bewirkten Auftrittsratenschwankungen andere Fehlereinflüsse dominieren (Mudelsee et al. 2003).

Eine weitere Annahme bei der statistischen Analyse ist die Unabhängigkeit der Ereignisse. Für die Elbehochwasser liegen die Datenpunkte Juni 2013 und August 2002 weit auseinander, und dazwischen gab es Phasen niedrigen Wasserstands, etwa im Hitzesommer 2003 ( Kap. 6); diese beiden Ereignisse können deshalb als unabhängig angesetzt werden. Wenn es dagegen, als hypothetisches Beispiel, nach dem Durchlaufen eines ersten Hochwasserscheitels nur eine kurzzeitige Senkung von 3 Tagen gegeben hätte und danach ein zweiter Scheitel aufgetreten wäre, dann gehörten die beiden Scheitel hydrologisch zusammen und würden als ein einzelnes Ereignis gewertet. Um die Unabhängigkeit der Ereignisse in einem Datensatz zu gewährleisten, sind deshalb oft detaillierte Studien der historischen Quellen oder der Abflusszeitreihen (• Abb. 11.1) notwendig (Mudelsee et al. 2003).

Das Analyseergebnis weicht wegen dieser Unsicherheiten von der wahren, jedoch unbekannten zeitabhängigen Auftrittsrate ab. Die typische Größe der Abweichungen, die durch die zufälligen Fluktuationen hervorgerufen werden, lässt sich statistisch bestimmen und in Form eines Unsicherheitsbandes angeben (- Abb. 11.2). Die typische Größe der Abweichungen, die auftreten, wenn die getroffenen Annahmen nicht zutreffen, lässt sich im Prinzip durch rechenintensive Wiederholungsanalysen bestimmen, nämlich durch Zugrundelegung variierter Annahmen.

Zum Beispiel simulierten Mudelsee et al. (2003) die für das Management von Hochwasser zur Verfügung stehende Reservoirgröße. Wenn um das Jahr 1900 - ab dem in nennenswerter Weise Reservoire für die Elbe gebaut wurden - und davor bereits der heutige Stauraum zur Verfügung gestanden hätte, dann hätte man durch geschicktes Hochwassermanagement den Hochwasserscheitel senken können. Mudelsee et al. (2003) erstellten derartige auf die Reservoirgröße korrigierte Hochwasserdaten für Elbe und Oder. Diese Korrektur zeigte nur einen geringen 
Einfluss auf die Trendbefunde. Des Weiteren erzeugten Mudelsee et al. (2003) simulierte Hochwasserdaten mithilfe eines Zufallsgenerators unter Zugrundelegung des Eichfehlers aus der Beziehung zwischen Wasserstand und Abfluss. Auch diese Simulationen zeigten nur einen geringen Einfluss auf die Trendbefunde.

Dieser Typ von Abweichungen wird als systematischer Fehler bezeichnet. Die Möglichkeiten derartiger Sensitivitätsanalysen sind jedoch insofern beschränkt, als das Wissen über Kausalfaktoren oder weitere Annahmen begrenzt ist.

\subsection{Unsicherheiten bei der statistischen Analyse von Projektionen in die Zukunft}

Um Wissen über die zukünftigen Hochwasserauftrittsraten zu erzeugen, werden Klimamodelle eingesetzt. Gegenwärtig wird mit Modellketten (Teil I) gearbeitet. Hierbei gibt ein räumlich gröber aufgelöstes globales Modell die Klimazustände am Rand eines feiner aufgelösten regionalen Modells (z. B. für Europa) vor. Der Output des regionalen Modells für die Variable „Niederschlag“ ist dann der Input des lokalen Impaktmodells, das die relevante Variable (Abfluss) in hoher Auflösung simuliert. An diese simulierte Abflusszeitreihe wird der Schwellenwert für die Definition extrem großer Ereignisse (• Abb. 11.1) angepasst. Die so simulierten Hochwasserereignisse werden mit der gleichen statistischen Methode analysiert wie die Hochwasserereignisse der Vergangenheit ( Abschn. 11.2).

Das Wissen über Hochwasserauftrittsraten in der Zukunft zeigt, wie das für die Vergangenheit, die beiden Unsicherheitstypen „zufällige Fluktuationen“ und „systematische Fehler“ (- Tab. 11.2). Die mit der Klimamodellierung zusammenhängenden Unsicherheiten treten nun an die Stelle der mit den Beobachtungen zusammenhängenden Unsicherheiten (Messwerte, historische Dokumente und Indikatorvariablen).

Bei globalen Klimamodellen bilden die anthropogenen Treibhausgasemissionen, welche die physikalischen Strahlungseigenschaften der Erde verändern, den wichtigsten Antrieb (Stocker et al. 2013); die Emissionen wirken sich stärker aus als natürliche Prozesse wie Schwankungen der Sonnenaktivität oder Vulkanaktivität. Ein Weg, der damit einhergehenden großen systematischen Unsicherheit zu begegnen, ist, bestimmte Emissionsszenarien vorzugeben und „Wenn-dann“-Rechnungen durchzuführen. Die hieraus hervorgehenden Klimaprojektionen decken eine Bandbreite an Emissionsszenarien ab (Teil I). Eine weitere Ursache systematischer Fehler bei globalen Klimamodellen liegt in der relativ groben räumlichen Auflösung. Dies erzwingt, für Prozesse, die auf kleinerer Raumskala ablaufen (z.B. Wolkenbildung), empirische physikalische Formeln, Parametrisierungen genannt, anzusetzen. Diese Formeln sind jedoch nicht exakt bekannt. Eine Möglichkeit, diese Unsicherheit zu quantifizieren, besteht in wiederholten Simulationen des globalen Klimas unter variierten Parametrisierungen (Allen 1999). Diese Vorgehensweise entspricht den Sensitivitätsanalysen, die für vergangene Klimazustände durchgeführt werden ( Abschn. 11.2). Die begrenzte Rechenkapazität erlaubt jedoch keine hohe Zahl an derartigen Modellsimulationen (- Tab. 11.2).
- Tab. 11.2 Unsicherheiten bei der statistischen Analyse von Hochwasser (zukünftiges Klima)

\begin{tabular}{l|l} 
Zufällige Fluktuationen & Systematische Fehler \\
\hline
\end{tabular}

Globales Klimamodell

Strahlungsantrieb (anthropogene Treibhausgasemissionen, natürliche Prozesse*)

Modellimplementierung (Nu- Modellformulierung merik)

Kurzfristige Fluktuationen (in den Variablen, die an das regionale Klimamodell übergeben werden)

Begrenzte Rechenkapazität

Regionales Klimamodell

Modellimplementierung (Numerik)

Modellformulierung

Kurzfristige Fluktuationen (Niederschlag)

Begrenzte Rechenkapazität

Lokales Impaktmodell

Modellimplementierung (Numerik)

Modellformulierung

Landnutzungsszenario

Wasserbauszenario

Begrenzte Rechenkapazität**

Statistische Methode

Begrenzte Datenverfügbarkeit

Schwellenwert für die Definition extrem großer Ereignisse

Begrenzte Rechenkapazität**

* Sonnenaktivität, Vulkanausbrüche

** Vernachlässigbar gering

Klima ist ein chaotisches System, in dem sich zwei hypothetische, zu einem Anfangszeitpunkt nahe beieinander liegende Klimazustände nach einer gewissen Zeit weit auseinander befinden. Um das chaotische Verhalten mit globalen Klimamodellen $\mathrm{zu}$ quantifizieren, werden wiederholte Simulationen unter variierten Anfangszuständen, sogenannte Ensemble-Simulationen, durchgeführt ( Kap. 5). Die begrenzte Rechenkapazität erlaubt auch hier keine hohe Anzahl an Ensembles (- Tab. 11.2).

Selbst wenn der zukünftige Strahlungsantrieb genau bekannt wäre, die Formulierung globaler Klimamodelle perfekt und der Anfangszustand exakt bestimmt, so würden doch zufällige Ergebnisabweichungen zwischen unterschiedlichen Modellimplementierungen resultieren. Dies liegt an der Wahl der Computerhardware und -software, die sich auf die Genauigkeit von Kommazahlen im Computer und der näherungsweisen numerischen Lösung von mathematischen Gleichungen auswirkt.

Bei den regionalen Klimamodellen besteht die wichtigste Ursache systematischer Fehler in der Modellformulierung ( Kap. 5). Durch die feinere räumliche Auflösung ist es mit regionalen Modellen möglich, zusätzliche (im Vergleich zu globa- 
len Modellen) biogeochemische Prozesse explizit mathematisch abzubilden; die Herausforderung ist jedoch, diese zusätzlichen Gleichungen realistisch zu formulieren. Und auch regionale Klimamodelle benötigen - unsichere - Parametrisierungen kleinskaliger Prozesse. Prinzipiell lassen sich diese Unsicherheiten wie bei den globalen Klimamodellen mithilfe von Simulationsrechnungen bestimmen.

Die relativen Anteile der einzelnen Unsicherheitsquellen an der gesamten Unsicherheit ändern sich mit der Zeit. Während momentan für die Jahre bis etwa 2030 die kurzfristigen chaotischen Klimafluktuationen den Hauptanteil ausmachen, überwiegen auf längerer Frist (bis 2100) die Unsicherheiten in den Treibhausgasemissionen und der Modellformulierung (Kirtman et al. 2013).

Auch bei den zufälligen Fluktuationen zeigen regionale Modelle Ähnlichkeiten zu den globalen Modellen ( $\mathbf{0}$ Tab. 11.2): numerische Aspekte der Modellimplementierung und begrenzte Rechenkapazität. Die chaotischen Eigenschaften auf der regionalen Skala betreffen nun die Klimavariable „Niederschlag“, die der Input für die Impaktmodelle ist.

Das lokale Impaktmodell ist ein hydrologisches Modell ( Kap. 10), das den Abfluss simuliert. Die Modellformulierung ist anfällig für systematische Fehler (z. B. Bodeneigenschaften). Von besonderer Bedeutung sind die nichtklimatischen Faktoren Landnutzungsänderung und Wasserbau (• Tab. 11.2). Wenn diese auch für das Hochwassergeschehen der mittleren Elbe in der Vergangenheit von vernachlässigbarem Einfluss waren $(\triangleright A b$ schn. 11.1), so mögen sie doch für die Zukunft oder für andere Flüsse (insbesondere kleine Einzugsgebiete) zu berücksichtigen sein. Anthropogenen Ursprungs wie der Faktor Treibhausgasemissionen, können Landnutzung und Wasserbau mithilfe von Szenarien analysiert werden. Die Streuung der Ergebnisse über diese Landnutzungs- oder Wasserbauszenarien ist ein grobes Maß der Analyseunsicherheiten. Während auch Impaktmodelle implementiert werden müssen, sind sie im Vergleich zu globalen oder regionalen Klimamodellen deutlich weniger komplex, und die begrenzte Rechenkapazität stellt hier kein Hindernis dar.

Für die statistische Analysemethode der Hochwasserdaten (ब Tab. 11.2) und deren Unsicherheitsquellen (hauptsächlich Datenverfügbarkeit und Schwellenwert) spielt es prinzipiell keine Rolle, ob die Daten die Zukunft betreffen (Modellkette) oder die Vergangenheit (Beobachtungen). Zur Wahl des Schwellenwerts im simulierten Abfluss für die Definition extrem großer Hochwasser (- Abb. 11.1): Um hier systematischen Fehlern vorzubeugen, müssen Simulationsergebnisse auch für die Vergangenheit vorliegen. Damit kann der Schwellenwert für die Modelldaten so eingestellt werden, dass im Überlappungszeitbereich Beobachtungen und Modellsimulationen gleich große Auftrittsraten zeigen.

Obwohl sich in den zurückliegenden Jahren mit steigender Rechenkapazität auch die Anzahl an Klimamodellsimulationen (das Produkt aus der Anzahl globaler Simulationen, der Anzahl regionaler Simulationen und der Anzahl an Impaktsimulationen) erhöht hat ( Kap. 5), sind es doch relativ wenige für die Anforderungen einer anspruchsvollen statistischen Analyse. Während für die einfache statistische Kenngröße „Mittelwert“ weniger Simulationen für eine belastbare Einschätzung benötigt werden, braucht es für die die Unsicherheit messende Kenngröße "Standardabweichung" mehr Simulationen, etwa 100-400 (Efron und Tibshirani 1993). Diese Größenordnung sollte in näherer Zukunft für die Teil-Modellketten (global-regional) betreibenden Institutionen machbar sein. Dazu darf die künftige, stark erhöhte Rechenkapazität jedoch nicht ausschließlich in eine feinere räumliche Auflösung der Modelle investiert werden.

\subsection{Unsicherheitsreduzierung durch intelligentes Fragen}

Vergangene und projizierte künftige Hochwasserereignisse können statistisch analysiert werden ( Abschn. 11.2 und 11.3). Ebenso kann der ursächliche Zusammenhang mit dem Klimawandel untersucht werden. Die mit diesen Analysen einhergehenden Unsicherheiten sind zum Teil beträchtlich (- Abb. 11.2). Wie lassen sie sich reduzieren? Für die Wissenschaft liegt der Schlüssel in der Auswahl des Schätzobjekts. Allgemeinverständlicher formuliert: Manche den Klimawandel betreffende Fragen lassen sich genauer als andere, in die gleiche Richtung zielende Fragen beantworten.

Als hypothetisches Beispiel seien die mit einer Modellkette simulierten Elbehochwasser für den Zeitraum von 1806 bis 2100 betrachtet. Das Schätzobjekt „zeitabhängige Auftrittsrate“ ist wegen der unterschiedlichen Unsicherheitsquellen ( $\mathbf{0}$ Tab. 11.2) nur sehr ungenau bekannt. Wenn man dagegen das Schätzobjekt „Auftrittsratenänderung (Vergangenheit/Zukunft)“ wählt, so wirken sich einige Unsicherheitsquellen, etwa die Wahl des Schwellenwerts für die Definition extrem großer Ereignisse, für die Vergangenheit gleich aus wie für die Zukunft, und die entsprechenden Unsicherheiten heben sich gegenseitig auf.

Generell sind vergleichende, Änderungen betreffende Fragestellungen „intelligenter" als absolute Fragestellungen. Auch die Unsicherheiten in der Modellformulierung ( $\mathbf{0}$ Tab. 11.2) lassen sich dadurch besser herauskürzen. Anderes Beispiel: Eine der "unintelligentesten“ Fragen wäre die nach einem Hochwasser in Meißen zu Ostern im Jahr 2084, also 300 Jahre nach dem großen Osterhochwasser an gleicher Stelle (Mudelsee et al. 2003).

Jedoch sind nicht alle alternativen Frageformulierungen für die Entscheidungsträger akzeptabel. Manchmal interessieren eben doch absolute Zahlen. Ein eng abgestimmtes Gespräch zwischen der Wissenschaft und den Entscheidungsträgern ist deshalb unerlässlich, um herauszufinden, was geht und welche Fragen die richtigen sind. Hierin liegt ein großes Potenzial.

\subsection{Kurz gesagt}

Extreme Wetter- und Klimaereignisse wie Hochwasser, Stürme oder Hitzewellen verursachten in Deutschland in der Vergangenheit beträchtliche ökonomische Schäden und Verluste an Menschenleben. Die Aufgabe, diese Ereignistypen in ihrem Auftreten zu quantifizieren, Trends darin nachzuweisen und eine kausale Zuschreibung in Bezug auf das Klima und andere Einflüsse vorzunehmen, stößt gegenwärtig auf erhebliche methodische Hindernisse und Unsicherheiten. Die Größe der Unsicherheiten lässt 
sich mithilfe von Simulations- und Sensitivitätsanalysen prinzipiell bestimmen, die Unsicherheiten selbst werden jedoch bestehen bleiben. Die methodischen Hindernisse lassen sich durch verbesserte statistische Algorithmen prinzipiell abtragen.

Wegen der Schwankungen im atmosphärischen System und wegen der Messfehler und Unsicherheiten in den Daten und Modellen weisen die Analyseergebnisse zwangsläufig Unsicherheiten auf. Während auf langen Zeitskalen aus physikalischen Gründen mit deutlichen, durch den Klimawandel hervorgerufenen Trends zu rechnen ist, stellen die Bestimmung der Auftrittsrate extremer Ereignisse und die Attribution weiterhin große wissenschaftliche Herausforderungen dar. Die Entscheidungen von Politik und Gesellschaft für dem Klimawandel begegnende Maßnahmen - betreffend Hochwasser und andere Extreme im Klimasystem - sind deshalb in einer Situation der Unsicherheit zu treffen. Intelligente, vergleichende Fragestellungen helfen hierbei, die Unsicherheiten zu reduzieren.

\section{Literatur}

Allen M (1999) Do-it-yourself climate prediction. Nature 401:642

Allen MR, Ingram WJ (2002) Constraints on future changes in climate and the hydrologic cycle. Nature 419:224-232

Belz JU, Busch N, Hammer M, Hatz M, Krahe P, Meißner D, Becker A, Böhm U, Gratzki A, Löpmeier F-J, Malitz G, Schmidt T (2013) Das Juni-Hochwasser des Jahres 2013 an den Bundeswasserstraßen - Ursachen und Verlauf Einordnung und fachliche Herausforderungen. Korresp Wasserwirtsch 6:624-634

Boucher O, Randall D, Artaxo P, Bretherton C, Feingold G, Forster P, Kerminen V-M, Kondo Y, Liao H, Lohmann U, Rasch P, Satheesh SK, Sherwood S, Stevens B, Zhang X-Y (2013) Clouds and aerosols. In: Stocker TF, Qin D, Plattner G-K, Tignor MMB, Allen SK, Boschung J, Nauels A, Xia Y, Bex V, Midgley PM (Hrsg) Climate change 2013: The physical science basis. Working group I contribution to the fifth assessment report of the Intergovernmental Panel on Climate Change. Cambridge University Press, Cambridge, S 571-657

Bronstert A (2003) The flood on the Elbe River in August 2002 and the efficiency of water retention in the Havel detention basins. Geophys Res Abstr 5:14648

Conradt T, Roers M, Schröter K, Elmer F, Hoffmann P, Koch H, Hattermann FF, Wechsung $F$ (2013) Vergleich der Extremhochwässer 2002 und $2013 \mathrm{im}$ deutschen Teil des Elbegebiets und deren Abflusssimulation durch SWIMlive. Hydrol Wasserbewirtsch 57:241-245

Czymzik M, Dulski P, Plessen B, von Grafenstein U, Naumann R, Brauer A (2010) A 450 year record of spring-summer flood layers in annually laminated sediments from Lake Ammersee (southern Germany). Water Resour Res 46:W11528. doi:10.1029/2009WR008360

Efron B, Tibshirani RJ (1993) An introduction to the bootstrap. Chapman \& Hall, New York

Hartmann DL, Klein Tank AMG, Rusticucci M, Alexander LV, Brönnimann S, Charabi YA-R, Dentener FJ, Dlugokencky EJ, Easterling DR, Kaplan A, Soden BJ, Thorne PW, Wild M, Zhai P (2013) Observations: Atmosphere and surface. In: Stocker TF, Qin D, Plattner G-K, Tignor MMB, Allen SK, Boschung J, Nauels A, Xia Y, Bex V, Midgley PM (Hrsg) Climate change 2013: The physical science basis. Working group I contribution to the fifth assessment report of the Intergovernmental Panel on Climate Change. Cambridge University Press, Cambridge, S 159-254

Helms M, Ihringer J, Nestmann F (2002) Analyse und Simulation des Abflussprozesses der Elbe. In: Nestmann F, Büchele B (Hrsg) Morphodynamik der Elbe. Institut für Wasserwirtschaft und Kulturtechnik. Universität Karlsruhe (TH), Karlsruhe, S 91-202

Hirabayashi Y, Mahendran R, Koirala S, Konoshima L, Yamazaki D, Watanabe S, Kim H, Kanae S (2013) Global flood risk under climate change. Nat Clim Change 3:816-821
Kirtman B, Power SB, Adedoyin AJ, Boer GJ, Bojariu R, Camilloni I, Doblas-Reyes F, Fiore AM, Kimoto M, Meehl G, Prather M, Sarr A, Schär C, Sutton R, Oldenborgh GJ van, Vecchi G, Wang H-J (2013) Near-term climate change: Projections and predictability. In: Stocker TF, Qin D, Plattner G-K, Tignor MMB, Allen SK, Boschung J, Nauels A, Xia Y, Bex V, Midgley PM (Hrsg) Climate change 2013: The physical science basis. Working group I contribution to the fifth assessment report of the Intergovernmental Panel on Climate Change. Cambridge University Press, Cambridge, S 953-1028

Königliche Elbstrombauverwaltung (1898) Der Elbstrom, sein Stromgebiet und seine wichtigsten Nebenflüsse, Bd 3.1. Reimer, Berlin

Mudelsee M (2014) Climate time series analysis: Classical statistical and bootstrap methods, 2. Aufl. Springer, Cham

Mudelsee M, Börngen M, Tetzlaff G, Grünewald U (2003) No upward trends in the occurrence of extreme floods in central Europe. Nature 425:166-169

Mudelsee M, Börngen M, Tetzlaff G, Grünewald U (2004) Extreme floods in central Europe over the past 500 years: Role of cyclone pathway "Zugstrasse Vb". J Geophys Res 109:D23101. doi:10.1029/2004JD005034

Mudelsee M, Deutsch M, Börngen M, Tetzlaff G (2006) Trends in flood risk of the river Werra (Germany) over the past 500 years. Hydrol Sci J 51:818-833

Ploeg RR van der, Schweigert P (2001) Elbe River flood peaks and postwar agricultural land use in East Germany. Naturwissenschaften 88:522-525

Stern N (2007) The economics of climate change: The Stern review. Cambridge University Press, Cambridge

Stocker TF, Qin D, Plattner G-K, Tignor MMB, Allen SK, Boschung J, Nauels A, Xia Y, Bex V, Midgley PM (2013) Climate change 2013: The physical science basis. Working group I contribution to the fifth assessment report of the Intergovernmental Panel on Climate Change. Cambridge University Press, Cambridge

Ward PJ, de Moel H, Aerts JCJH (2011) How are flood risk estimates affected by the choice of return-periods? Nat Hazards Earth Syst Sci 11:3181-3195

Open Access Dieses Kapitel wird unter der Creative Commons Namensnennung 4.0 International Lizenz (http://creativecommons.org/ licenses/by/4.0/deed.de) veröffentlicht, welche die Nutzung, Vervielfältigung, Bearbeitung, Verbreitung und Wiedergabe in jeglichem Medium und Format erlaubt, sofern Sie den/die ursprünglichen Autor(en) und die Quelle ordnungsgemäß nennen, einen Link zur Creative Commons Lizenz beifügen und angeben, ob Änderungen vorgenommen wurden.

Etwaige Abbildungen oder sonstiges Drittmaterial unterliegen ebenfalls der genannten Creative Commons Lizenz, sofern sich aus der Abbildungslegende oder der Quellreferenz nichts anderes ergibt. Sofern solches Drittmaterial nicht unter der genannten Creative Commons Lizenz steht, ist eine Vervielfältigung, Bearbeitung oder öffentliche Wiedergabe nur mit vorheriger Zustimmung des betreffenden Rechteinhabers oder auf der Grundlage einschlägiger gesetzlicher Erlaubnisvorschriften zulässig. 